\title{
Optimal Transmission Strategy and Explicit Capacity Region for Broadcast Z Channels
}

\author{
Bike Xie, Student Member, IEEE, Miguel Griot, Student Member, IEEE, \\ Andres I. Vila Casado, Student Member, IEEE, and Richard D. Wesel, Senior Member, IEEE
}

\begin{abstract}
This paper provides an explicit expression for the capacity region of the two-user broadcast $Z$ channel and proves that the optimal boundary can be achieved by independent encoding of each user. Specifically, the information messages corresponding to each user are encoded independently and the OR of these two encoded streams is transmitted. Nonlinear turbo codes that provide a controlled distribution of ones and zeros are used to demonstrate a low-complexity scheme that operates close to the optimal boundary.
\end{abstract}

Index Terms-Broadcast channel, broadcast $\mathrm{Z}$ channel, capacity region, nonlinear turbo codes, turbo codes.

\section{INTRODUCTION}

D EGRADED broadcast channels were first studied by Cover in [1] and a formulation of the capacity region was established in [2], [3], and [4]. Superposition encoding is the key idea to achieve the optimal boundary of the capacity region for degraded broadcast channels [5]. With superposition encoding for degraded broadcast channels, the data sent to the user with the most degraded channel is encoded first. Given the encoded bits for that user, an appropriate codebook for the second most degraded channel user is selected, and so forth. Hence, superposition encoding is, in general, a joint encoding scheme. However, combining independently encoded streams, one for each user, is an optimal scheme for some broadcast channels including broadcast Gaussian channels [1] and broadcast binary-symmetric channels [1], [2].

Successive decoding is a natural decoding scheme for superposition encoding [1], [2], [5]. With successive decoding for degraded broadcast channels, each receiver first decodes the data sent to the user with the most degraded channel. Conditioning on the decoded data for that user, each receiver determines the codebook for the user with the second most degraded channel and decodes that data, and so forth until the desired user's data is decoded. The performance of successive decoding for degraded broadcast channels is very close to optimal decoding under normal operating conditions.

Manuscript received August 22, 2007; revised June 7, 2008. Published August 27, 2008 (projected). This work was supported by the Defence Advanced Research Project Agency SPAWAR Systems Center, San Diego, California under Grant N66001-02-1-8938. This paper was presented in part at the Information Theory Workshop, Lake Tahoe, CA, September 2007.

The authors are with the Electrical Engineering Department, University of California, Los Angeles, CA 90095 USA (e-mail: xbk@ee.ucla.edu; mgriot@ee.ucla.edu; avila@ee.ucla.edu; wesel@ee.ucla.edu).

Communicated by H. Yamamoto, Associate Editor for Shannon Theory.

Color versions of Figures 7 and 14 in this paper are available online at http:// ieeexplore.ieee.org.

Digital Object Identifier 10.1109/TIT.2008.928298

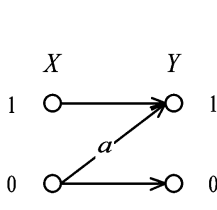

(a)

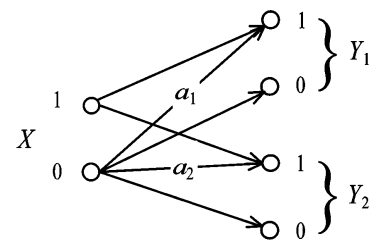

(b)
Fig. 1. (a) $\mathrm{Z}$ channel. (b) Broadcast $\mathrm{Z}$ channel.

Turbo codes [6] and low-density parity-check (LDPC) codes [7] perform close to the Shannon limit. LDPC and turbo coding approach for broadcast channels were studied in [8] and [9], respectively. In [8], LDPC codes provided reliable transmission over two-user broadcast channels with additive white Gaussian noise (AWGN) and fading known at the receiver only. In [9], a superposition turbo coding scheme performs within $1 \mathrm{~dB}$ of the capacity region boundary for broadcast Gaussian channels. Both of these approaches are designed specifically for broadcast Gaussian channels and used linear codes. For multi-user binary adder channels, nonlinear trellis codes were studied and designed in [10].

The $\mathrm{Z}$ channel is the binary-asymmetric channel shown in Fig. 1(a). The capacity of the $\mathrm{Z}$ channel was studied in [11]. Nonlinear trellis codes were designed to maintain a low ones density for the $\mathrm{Z}$ channel in [12] and [14] and parallel concatenated nonlinear turbo codes were designed for the $\mathrm{Z}$ channel in [13]. This paper focuses on the study of the two-user broadcast $\mathrm{Z}$ channel $X \rightarrow Y_{1}, Y_{2}$ shown in Fig. 1(b). This paper provides an explicit expression of the capacity region for the two-user broadcast $\mathrm{Z}$ channel and shows that independent encoding with successive decoding can achieve the boundary of this capacity region.

This paper is organized as follows. Section II introduces definitions and notation for broadcast channels. Section III provides the explicit expression of the capacity region for the two-user broadcast $\mathrm{Z}$ channel and proves that independent encoding can achieve the optimal boundary of the capacity region. Section IV presents nonlinear-turbo codes designed to achieve the optimal boundary, and Section V provides the simulation results. Section VI delivers the conclusions.

\section{DEFINITIONS AND PRELIMINARIES}

\section{A. Degraded Broadcast Channels}

The general representation of a discrete memoryless broadcast channel is given in Fig. 2. A single signal $X$ is broadcast to $M$ users through $M$ different channels $A_{1}, \ldots, A_{M}$. If $p\left(y_{i}, y_{i+1} \mid x\right)=p\left(y_{i} \mid x\right) p\left(y_{i+1} \mid y_{i}\right)$, then channel $A_{i+1}$ is 


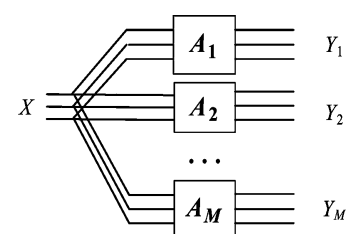

Fig. 2. Broadcast channel.

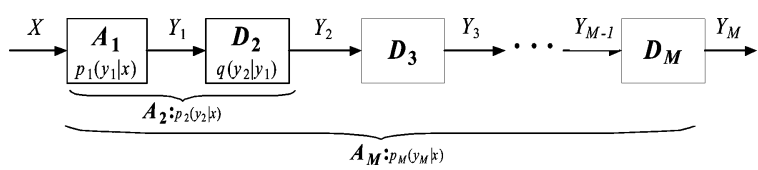

Fig. 3. Physically degraded broadcast channel.

a physically degraded version of channel $A_{i}$ (and thus the broadcast channel $X \rightarrow Y_{i}, Y_{i+1}$ is physically degraded) [5]. A physically degraded broadcast channel with $M$ users is shown in Fig. 3. Since each user decodes its received signal without collaboration, only the marginal transition probabilities $p\left(y_{1} \mid x\right), p\left(y_{2} \mid x\right), \ldots, p\left(y_{M} \mid x\right)$ of the component channels $A_{1}, A_{2}, \ldots, A_{M}$ affect receiver performance. Hence, the stochastically degraded broadcast channel is defined in [2] and [5] as follows.

Let $A_{i}$ be a channel with input alphabet $\mathcal{X}$, output alphabet $\mathcal{Y}_{i}$, and transition probability $p_{i}\left(y_{i} \mid x\right)$. Let $A_{i+1}$ be another channel with the same input alphabet $\mathcal{X}$, output alphabet $\mathcal{Y}_{i+1}$, and transition probability $p_{i+1}\left(y_{i+1} \mid x\right)$. $A_{i+1}$ is a stochastically degraded version of $A_{i}$ if there exists a transition probability $q\left(y_{i+1} \mid y_{i}\right)$ such that

$$
p_{i+1}\left(y_{i+1} \mid x\right)=\sum_{y_{i} \in \mathcal{Y}_{i}} q\left(y_{i+1} \mid y_{i}\right) p_{i}\left(y_{i} \mid x\right) .
$$

A broadcast channel with receivers $Y_{1}, Y_{2} \ldots, Y_{M}$ is a stochastically degraded broadcast channel if every component channel $A_{i}$ is a stochastically degraded version of $A_{i-1}$ for all $i=2, \ldots, M$ [2]. Since the marginal transition probabilities $p\left(y_{1} \mid x\right), p\left(y_{2} \mid x\right), \ldots, p\left(y_{M} \mid x\right)$ completely determine a stochastically degraded broadcast channel, we can model any stochastically degraded broadcast channel as a physically degraded broadcast channel with the same marginal transition probabilities.

Theorem 1 ([2] and [4]): The capacity region for the twouser stochastically degraded broadcast channel $X \rightarrow Y_{1} \rightarrow Y_{2}$ is the convex hull of the closure of all $\left(R_{1}, R_{2}\right)$ satisfying

$$
R_{2} \leq I\left(X_{2} ; Y_{2}\right) \quad R_{1} \leq I\left(X ; Y_{1} \mid X_{2}\right)
$$

for some joint distribution $p\left(x_{2}\right) p\left(x \mid x_{2}\right) p\left(y_{1}, y_{2} \mid x\right)$, where the auxiliary random variable $X_{2}{ }^{1}$ has cardinality bounded by $\left|\mathcal{X}_{2}\right| \leq \min \left\{|\mathcal{X}|,\left|\mathcal{Y}_{1}\right|,\left|\mathcal{Y}_{2}\right|\right\}$.

\section{B. The Broadcast Z Channel}

The $\mathrm{Z}$ channel, shown in Fig. 1(a), is a binary-asymmetric channel with the transition probability matrix

$$
T=\left[\begin{array}{cc}
1 & \alpha \\
0 & 1-\alpha
\end{array}\right]
$$

${ }^{1} U$ was used as the auxiliary random variable in [2], [4]. In this paper, we use $X_{2}$ instead of $U$ because the auxiliary random variable corresponds to the second user's encoded stream.

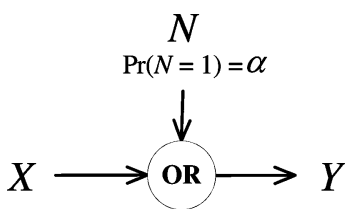

Fig. 4. OR operation view of $\mathrm{Z}$ channel.

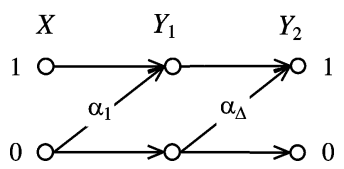

Fig. 5. Physically degraded broadcast $\mathrm{Z}$ channel.

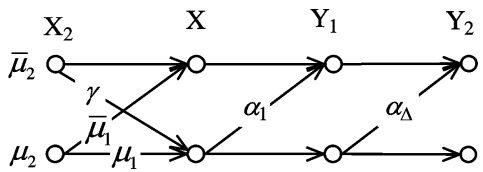

Fig. 6. Information theoretic diagram of the system.

where $0 \leq \alpha \leq 1$. If symbol 1 is transmitted, symbol 1 is received with probability 1 . If symbol 0 is transmitted, symbol 1 is received with probability $\alpha$ and symbol 0 is received with probability $1-\alpha$. We can model the $\mathrm{Z}$ channel as the OR operation of the channel input $X$ and Bernoulli noise $N$ with parameter $\alpha$ as shown in Fig. 4. In an OR multiple-access channel (MAC), each user appears to transmit over a $\mathrm{Z}$ channel when the other users are treated as noise [13]. Thus, in an OR network with multiple transmitters and multiple receivers, each transmitter transmitting to more than one receiver sees a broadcast $\mathrm{Z}$ channel if other transmitters transmitting to those receivers are treated as noise. The two-user broadcast $\mathrm{Z}$ channel with the marginal transition probability matrices

$$
T_{1}=\left[\begin{array}{cc}
1 & \alpha_{1} \\
0 & 1-\alpha_{1}
\end{array}\right] \quad T_{2}=\left[\begin{array}{cc}
1 & \alpha_{2} \\
0 & 1-\alpha_{2}
\end{array}\right]
$$

is shown in Fig. 1, where $0 \leq \alpha_{1} \leq \alpha_{2} \leq 1$. Because broadcast $\mathrm{Z}$ channels are stochastically degraded, we can model any broadcast $\mathrm{Z}$ channel as a physically degraded broadcast $\mathrm{Z}$ channel as shown in Fig. 5, where

$$
\alpha_{\Delta}=\frac{\alpha_{2}-\alpha_{1}}{1-\alpha_{1}} .
$$

\section{OPtimal TRANSMisSion STRATEGy FOR THE Two-USER BROADCAST Z CHANNEL}

Since the broadcast $\mathrm{Z}$ channel is stochastically degraded, its capacity region can be obtained directly from Theorem 1 . The capacity region for the broadcast $\mathrm{Z}$ channel $X \rightarrow Y_{1} \rightarrow Y_{2}$ as shown in Fig. 6 is the convex hull of the closure of all $\left(R_{1}, R_{2}\right)$ satisfying

$$
\begin{aligned}
R_{2} \leq & I_{2}=I\left(X_{2} ; Y_{2}\right) \\
= & H\left(\left(\bar{\mu}_{2} \gamma+\mu_{2} \mu_{1}\right)\left(1-\alpha_{2}\right)\right) \\
& -\bar{\mu}_{2} H\left(\gamma\left(1-\alpha_{2}\right)\right)-\mu_{2} H\left(\mu_{1}\left(1-\alpha_{2}\right)\right) \\
R_{1} \leq & I_{1}=I\left(X ; Y_{1} \mid X_{2}\right) \\
= & \bar{\mu}_{2}\left(H\left(\gamma\left(1-\alpha_{1}\right)\right)-\gamma H\left(1-\alpha_{1}\right)\right) \\
& +\mu_{2}\left(H\left(\mu_{1}\left(1-\alpha_{1}\right)\right)-\mu_{1} H\left(1-\alpha_{1}\right)\right)
\end{aligned}
$$




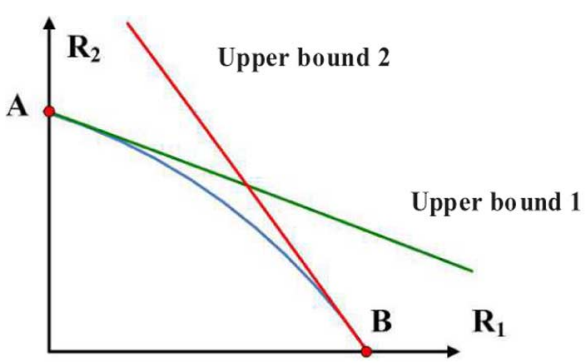

(a)

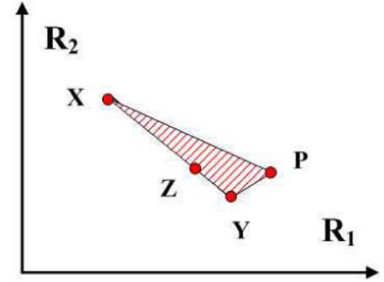

(b)

Fig. 7. (a) The capacity region and two upper bounds. (b) Point $Z$ cannot be on the boundary of the capacity region.

for some probabilities $\mu_{1}, \mu_{2}, \gamma$, where $\mu_{1}=\operatorname{Pr}\left(x=0 \mid x_{2}=\right.$ $0), \mu_{2}=\operatorname{Pr}\left(x_{2}=0\right), \gamma=\operatorname{Pr}\left(x=0 \mid x_{2}=1\right), H(\cdot)$ is the binary entropy function, $\bar{\mu}_{1}=1-\mu_{1}, \bar{\mu}_{2}=1-\mu_{2}$ and

$$
\alpha_{2}=\operatorname{Pr}\left\{y_{2}=1 \mid x=0\right\}=1-\left(1-\alpha_{1}\right)\left(1-\alpha_{\Delta}\right) .
$$

Each particular choice of $\left(\mu_{1}, \mu_{2}, \gamma\right)$ in Fig. 6 specifies a particular transmission strategy and a rate pair $\left(I_{1}, I_{2}\right)$. The optimal boundary of a capacity region is the set of all Pareto optimal points $\left(I_{1}, I_{2}\right)$, for which it is impossible to increase rate $I_{1}$ without decreasing rate $I_{2}$ or vice versa. A transmission strategy is optimal if and only if it achieves a rate pair point on the optimal boundary. We call a set of transmission strategies sufficient if all rate pairs on the optimal boundary can be achieved by using these strategies and time sharing. Furthermore, a set of transmission strategies is strongly sufficient if these strategies can achieve all rate pairs on the optimal boundary without using time sharing. Equations (4) and (5) give a set of pentagons that yield the capacity region through their convex hull, but do not explicitly show the optimal transmission strategies or derive the boundary of the capacity region.

\section{A. Optimal Transmission Strategies}

The following theorem identifies a set of optimal transmission strategies and provides an explicit expression of the boundary of the capacity region.

Theorem 2: For a broadcast $\mathrm{Z}$ channel with $0<\alpha_{1}<\alpha_{2}<1$, the set of the optimal transmission strategies $\left(\mu_{1}, \mu_{2}, \gamma\right)$, which satisfy

$$
\begin{gathered}
\gamma=0 \\
\frac{1}{\left(1-\alpha_{1}\right)\left(e^{H\left(1-\alpha_{1}\right) /\left(1-\alpha_{1}\right)}+1\right)} \leq \mu_{1} \leq 1
\end{gathered}
$$

and

$$
\begin{aligned}
(H & \left.\left(\mu_{1}\left(1-\alpha_{1}\right)\right)-\mu_{1} H\left(1-\alpha_{1}\right)\right) \cdot \ln \left(1-\mu_{1}\left(1-\alpha_{2}\right)\right) \\
= & \left(H\left(\mu_{1}\left(1-\alpha_{2}\right)\right)-\mu_{1}\left(1-\alpha_{2}\right) \ln \frac{1-\mu_{2} \mu_{1}\left(1-\alpha_{2}\right)}{\mu_{2} \mu_{1}\left(1-\alpha_{2}\right)}\right) \\
& \cdot \ln \left(1-\mu_{1}\left(1-\alpha_{1}\right)\right)
\end{aligned}
$$

are strongly sufficient. In other words, all rate pairs on the optimal boundary of the capacity region can be achieved by using exactly the transmission strategies described in (7)-(9) without the need of time sharing. Furthermore, applying (7)-(9) to (4) and (5) yields an explicit expression of the optimal boundary of the capacity region.

Before proving Theorem 2, we present and prove some preliminary results. From (4) and (5), we can see that the transmission strategies $\left(\mu_{1}, \mu_{2}, \gamma\right)$ and $\left(\gamma, 1-\mu_{2}, \mu_{1}\right)$ have the same transmission rate pairs. Therefore, we assume $\gamma \leq \mu_{1}$ in the rest of the section without loss of generality.

Theorem 3: For a broadcast Z channel with $0<\alpha_{1}<\alpha_{2}<$ 1 , any transmission strategy $\left(\mu_{1}, \mu_{2}, \gamma\right)$ with $0<\mu_{2}<1,0<$ $\gamma<\mu_{1}$ is not optimal.

The proof is given in Appendix A.

Corollary 1: The set of all the transmission strategies with $\gamma=0$ is sufficient for any broadcast $\mathrm{Z}$ channel with $0<\alpha_{1}<$ $\alpha_{2}<1$.

Proof: From Theorem 3, we know that the transmission strategy $\left(\mu_{1}, \mu_{2}, \gamma\right)$ is optimal only if at least one of these four equations $\mu_{2}=0, \mu_{2}=1, \gamma=\mu_{1}, \gamma=0$ is true. Hence the set of all the transmission strategies with $\mu_{2}=0, \mu_{2}=1$, $\gamma=\mu_{1}$ or $\gamma=0$ is sufficient. When $\mu_{2}=0, \mu_{2}=1$ or $\gamma=\mu_{1}$, the transmission rate for the second user, $I_{2}$ in (4), is zero. This optimal rate pair is the point $B$ in Fig. 7(a). Since this point can also be achieved by the transmission strategy with $\gamma=0$, $\mu_{2}=1$ and $\mu_{1}=\arg \max \left(H\left(x\left(1-\alpha_{1}\right)\right)-x H\left(1-\alpha_{1}\right)\right)$, all the optimal rate pairs on the optimal boundary of the capacity region can be achieved by using the transmission strategies with $\gamma=0$ and time sharing. Thus, the set of all the transmission strategies with $\gamma=0$ is sufficient.

Q.E.D.

From Corollary 1, we can set $\gamma=0$ in Fig. 6 without losing any part of the capacity region and so the designed virtual channel $X_{2} \rightarrow X$ is a $\mathrm{Z}$ channel. Since we can consider the output of a $\mathrm{Z}$ channel as the OR operation of two Bernoulli random variables, an independent encoding scheme that works well for the broadcast $\mathrm{Z}$ channel will be introduced later in this paper.

Applying $\gamma=0$ to (4) and (5) yields

$$
\begin{aligned}
& R_{2} \leq I_{2}=H\left(\mu_{2} \mu_{1}\left(1-\alpha_{2}\right)\right)-\mu_{2} H\left(\mu_{1}\left(1-\alpha_{2}\right)\right) \\
& R_{1} \leq I_{1}=\mu_{2} H\left(\mu_{1}\left(1-\alpha_{1}\right)\right)-\mu_{2} \mu_{1} H\left(1-\alpha_{1}\right) .
\end{aligned}
$$

By Corollary 1, the capacity region is the convex hull of the closure of all rate pairs $\left(R_{1}, R_{2}\right)$ satisfying (10) and (11) for some probability $\mu_{1}, \mu_{2}$. However, not all transmission strategies of $\left(\mu_{1}, \mu_{2}, \gamma=0\right)$ achieve the optimal boundary of the 
capacity region. Since any optimal transmission strategy maximizes $I_{1}+\lambda I_{2}$ for some nonnegative $\lambda$, we solve the optimization problem of maximizing $I_{1}+\lambda I_{2}$ for any fixed $\lambda \geq 0$ in order to find the constraints on $\mu_{1}$ and $\mu_{2}$ for optimal transmission strategies. Theorem 4 provides the solution to this maximization problem.

Theorem 4: The optimal solution to the maximization problem

$$
\begin{array}{cl}
\operatorname{maximize} & I_{1}+\lambda I_{2} \\
\text { subject to } & I_{2}=H\left(\mu_{2} \mu_{1}\left(1-\alpha_{2}\right)\right)-\mu_{2} H\left(\mu_{1}\left(1-\alpha_{2}\right)\right) \\
& I_{1}=\mu_{2} H\left(\mu_{1}\left(1-\alpha_{1}\right)\right)-\mu_{2} \mu_{1} H\left(1-\alpha_{1}\right) \\
& 0 \leq \mu_{2} \leq 1, \quad 0 \leq \mu_{1} \leq 1
\end{array}
$$

is unique and it is given below for any fixed $\lambda \geq 0$.

Define

$$
\varphi(x)=\frac{\ln \left(1-\left(1-\alpha_{1}\right) x\right)}{\ln \left(1-\left(1-\alpha_{2}\right) x\right)}
$$

and

$$
\psi(x)=\frac{1}{x e^{H(x) / x}+x} .
$$

Case 1: If $0 \leq \lambda \leq \varphi\left(\psi\left(1-\alpha_{1}\right)\right)$, then the optimal solution is $\mu_{2}^{*}=1, \mu_{1}^{*}=\psi\left(1-\alpha_{1}\right)$, which satisfies (8) and (9), and the corresponding rate pair is $I_{1}^{*}=H\left(\mu_{1}^{*}\left(1-\alpha_{1}\right)\right)-\mu_{1}^{*} H\left(1-\alpha_{1}\right)$, $I_{2}^{*}=0$.

Case 2: If $\lambda \geq \varphi(1)$, then the optimal solution is $\mu_{2}^{*}=$ $\psi\left(1-\alpha_{2}\right), \mu_{1}^{*}=1$, which also satisfies (8) and (9), and the corresponding rate pair is $I_{1}^{*}=0, I_{2}^{*}=H\left(\mu_{2}^{*}\left(1-\alpha_{2}\right)\right)-$ $\mu_{2}^{*} H\left(1-\alpha_{2}\right)$.

Case 3: If $\varphi\left(\psi\left(1-\alpha_{1}\right)\right)<\lambda<\varphi(1)$, then the optimal solution given below also satisfies (8) and (9)

$$
\mu_{1}^{*}=\varphi^{-1}(\lambda)=\frac{e^{\lambda}-1}{e^{\lambda}\left(1-\alpha_{2}\right)-\left(1-\alpha_{1}\right)}
$$

and

$$
\begin{aligned}
& \left(H\left(\mu_{1}^{*}\left(1-\alpha_{1}\right)\right)-\mu_{1}^{*} H\left(1-\alpha_{1}\right)\right) \cdot \ln \left(1-\mu_{1}^{*}\left(1-\alpha_{2}\right)\right) \\
& =\left(H\left(\mu_{1}^{*}\left(1-\alpha_{2}\right)\right)-\mu_{1}^{*}\left(1-\alpha_{2}\right) \ln \frac{1-\mu_{2}^{*} \mu_{1}^{*}\left(1-\alpha_{2}\right)}{\mu_{2}^{*} \mu_{1}^{*}\left(1-\alpha_{2}\right)}\right) \\
& \quad \cdot \ln \left(1-\mu_{1}^{*}\left(1-\alpha_{1}\right)\right) .
\end{aligned}
$$

The proof is given in Appendix B. Combining Case 1,2 and 3 , we conclude that $\left(\mu_{1}, \mu_{2}\right)$ is a maximizer of (12) if and only if the pair $\left(\mu_{1}, \mu_{2}\right)$ satisfies (8) and (9). In other words, if $\left(\mu_{1}, \mu_{2}\right)$ doesn't satisfy (8) or (9), $\left(\mu_{1}, \mu_{2}\right)$ cannot be a maximizer of (12), and thus the transmission strategy $\left(\mu_{1}, \mu_{2}, \gamma=0\right)$ is not optimal. Since the set of the transmission strategies with $\gamma=$ 0 is sufficient by Corollary 1, the set of all the transmission strategies satisfying (7)-(9) is also sufficient. Therefore the capacity region is the convex hull of the closure of all rate pairs
( $\left.R_{1}, R_{2}\right)$ satisfying (10) and (11) for some $\mu_{1}, \mu_{2}$ which satisfy (8) and (9).

A sketch of the capacity region is shown with two upper bounds in Fig. 7(a). From Case 1 in Theorem 4, the point $B$ corresponds to the largest transmission rate for the first user. The first upper bound is the tangent of the capacity region at the point $B$, and its slope is $-1 / \varphi\left(\psi\left(1-\alpha_{1}\right)\right)$. From Case 2, the point $A$ provides the largest transmission rate for the second user. The second upper bound is the tangent of the capacity region at the point $A$, and its slope is $-1 / \varphi(1)$. Case 3 gives us the optimal boundary of the capacity region except the points $A$ and $B$.

Given $\alpha_{1}$ and $\alpha_{2}$, which completely describe a two-user degraded broadcast $\mathrm{Z}$ channel, the optimal boundary of the capacity region can be explicitly described by (8)-(11). For any $\mu_{1}$ in the range of (8), the value of the unique associated $\mu_{2}$ follows from (9). The curve of the optimal boundary of the capacity region is then the set of $\left(I_{1}, I_{2}\right)$ pairs satisfying (10) and (11) for these $\mu_{1}$ and associated $\mu_{2}$. For example, for $\alpha_{1}=0.15$ and $\alpha_{2}=0.6$, the range of optimal $\mu_{1}$ values is $0.445 \leq \mu_{1} \leq 1$, the range of optimal $\mu_{2}$ values implied by (9) is $0.392 \leq \mu_{2} \leq 1$, and the associated capacity region boundary is plotted in Fig. 13 .

Now we prove Theorem 2. Since we have proved that the set of all the transmission strategies satisfying (7)-(9) is sufficient, we only need to show that any rate pair on the optimal boundary of the capacity region can be achieved without using time sharing.

Proof by Contradiction: Suppose the point $Z$ in Fig. 7(b) is on the optimal boundary of the capacity region for the broadcast $\mathrm{Z}$ channel and this point can only be achieved by time sharing of the points $X$ and $Y$, which can be directly achieved by using transmission strategies satisfying (7)-(9). Clearly, the slope of the line segment $X Y$ is neither zero nor minus infinity. Denote $-k, 0<k<\infty$ as the slope of $X Y$. The points $X$ and $Y$ provide the same value of $I_{1}+\frac{1}{k} I_{2}$. By Theorem 4, the optimal solution to the maximization problem of $\max \left(I_{1}+\lambda I_{2}\right)$ is unique, and so neither $X$ nor $Y$ maximizes $\left(I_{1}+\frac{1}{k} I_{2}\right)$. Thus, there exists an achievable point $P$ such that this point is on the right upper side of the line $X Y$. Since and the triangle $\triangle X Y P$ is in the capacity region, the point $Z$ must not be on the optimal boundary of the capacity region (contradiction).

Q.E.D.

\section{B. Independent Encoding Scheme}

The communication system for the two-user broadcast $\mathrm{Z}$ channel is shown in Fig. 8. In a general scheme, the transmitter jointly encodes the independent messages $W_{1}$ and $W_{2}$, which is potentially too complex to implement. Theorem 2 demonstrates that there exists an independent encoding scheme which achieves the optimal boundary of the capacity region. Since $\gamma=0$ is strongly sufficient, the designed channel $X_{2} \rightarrow X$ is a $\mathrm{Z}$ channel. Thus, the broadcast signal $X$ can be constructed as the OR of two Bernoulli random variables $X_{1}$ and $X_{2}$. This construction of $X$ is an independent encoding scheme. The system diagram of the independent encoding scheme is shown in Fig. 9. First the messages $W_{1}$ and $W_{2}$ are encoded separately and independently. $X_{1}$ and $X_{2}$ are two binary random variables with $\operatorname{Pr}\left\{X_{j}=1\right\}=\bar{\mu}_{j}$ and $\operatorname{Pr}\left\{X_{j}=0\right\}=\mu_{j}$, where 


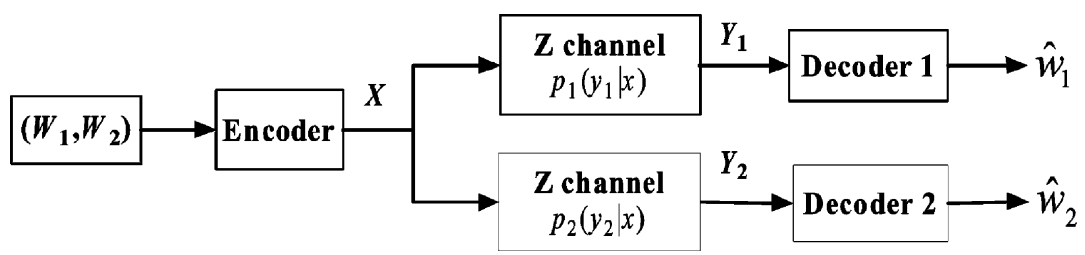

Fig. 8. Communication system for two-user broadcast $\mathrm{Z}$ channels.

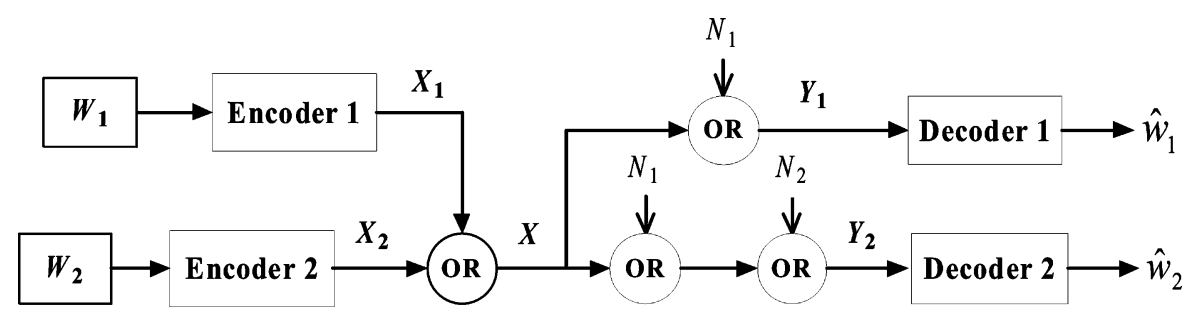

Fig. 9. Optimal transmission strategy for broadcast $\mathrm{Z}$ channels.

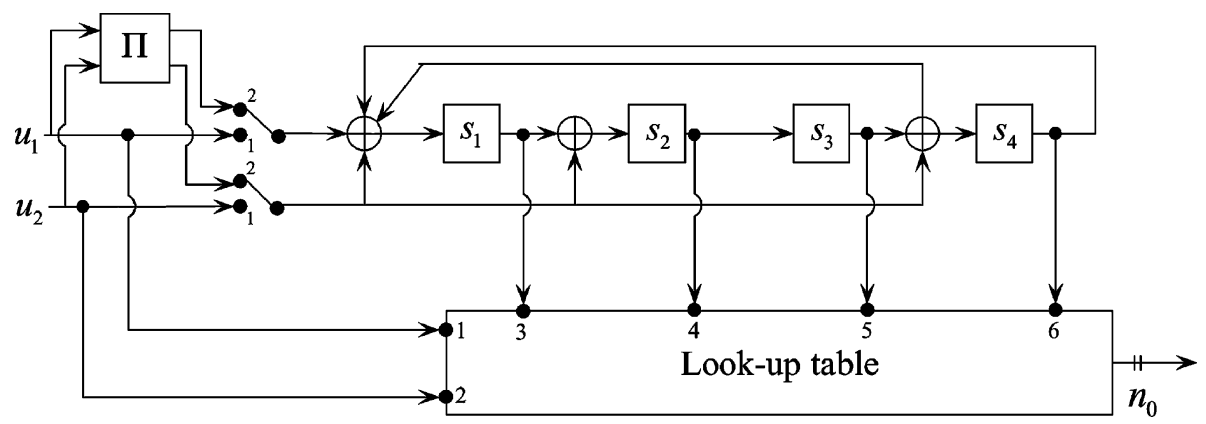

Fig. 10. 16-state nonlinear turbo code structure, with $k_{0}=2$ input bits per trellis section.

$\bar{\mu}_{j}+\mu_{j}=1$ for $j=1,2$. The transmitter broadcasts $X$, which is the OR of $X_{1}$ and $X_{2}$. From Theorem 2, this independent encoding scheme with any choice of $\left(\mu_{1}, \mu_{2}\right)$ satisfying (8) and (9) achieves a rate pair $\left(I_{1}, I_{2}\right)$ arbitrarily close to the optimal boundary of the capacity region if the codes for $X_{1}$ and $X_{2}$ are properly chosen and have sufficiently large block lengths.

\section{NONLINEAR-TURBo CODES FOR THE TWO-USER BROADCAST Z CHANNEL}

In this section we show a practical implementation of the transmission strategy for the two-user broadcast $\mathrm{Z}$ channel. As proved in Section III, the optimal boundary is achieved by transmitting the OR of the encoded data of each user, provided that the density of ones of each of these encoded streams is chosen properly. Hence, a family of codes that provides a controlled density of ones is required. We use the nonlinear turbo codes, introduced in [13], to provide the needed controlled density of ones. Nonlinear turbo codes are parallel concatenated trellis codes with $k_{0}$ input bits and $n_{0}$ output bits per trellis section. A look-up table assigns the output label for each branch of the trellis so that the required ones density is achieved. Each constituent encoder for the turbo code in this paper is a 16-state trellis code with $k_{0}=2$ and the trellis structure shown in Fig. 10. The output labels are assigned via a constrained search that provides the required ones density for each case, using the tools presented in [13] for the Z Channel. The output labels for the codes with rate pair $\left(R_{1}=1 / 6, R_{2}=1 / 6\right)$, which is simulated on a broadcast $\mathrm{Z}$ channel with $\alpha_{1}=0.15, \alpha_{2}=0.6$, are listed in Table I.
TABLE I

LABELING FOR CONSTituent TRELlis Codes. RATES $R_{1}=1 / 6, R_{2}=1 / 6$ ROWS REPRESENT THE STATE $s_{1} s_{2} s_{3} s_{4}$, COLUMNS REPRESENT THE INPUT $u_{1} u_{2}$. LABELING IN OCTAL NOTATION

\begin{tabular}{|c||c|c|c|c|}
\hline \multicolumn{5}{|c|}{ User 1 } \\
\hline \multicolumn{1}{|c||}{ state } & \multicolumn{4}{c|}{ input } \\
\cline { 2 - 5 } & 00 & 01 & 10 & 11 \\
\hline 0000 & 40 & 20 & 10 & 04 \\
\hline 0001 & 20 & 40 & 04 & 10 \\
\hline 0010 & 10 & 04 & 02 & 01 \\
\hline 0011 & 04 & 10 & 01 & 02 \\
\hline 0100 & 02 & 01 & 40 & 20 \\
\hline 0101 & 01 & 02 & 20 & 40 \\
\hline 0110 & 42 & 21 & 14 & 05 \\
\hline 0111 & 21 & 42 & 05 & 14 \\
\hline 1000 & 01 & 02 & 04 & 10 \\
\hline 1001 & 02 & 01 & 10 & 04 \\
\hline 1010 & 04 & 10 & 20 & 40 \\
\hline 1011 & 10 & 04 & 40 & 20 \\
\hline 1100 & 05 & 14 & 21 & 42 \\
\hline 1101 & 14 & 05 & 42 & 21 \\
\hline 1110 & 20 & 40 & 01 & 02 \\
\hline 1111 & 40 & 20 & 02 & 01 \\
\hline
\end{tabular}

\begin{tabular}{|c||c|c|c|c|}
\hline \multicolumn{5}{|c||}{ User 2 } \\
\hline \multicolumn{1}{|c||}{ state } & \multicolumn{4}{c|}{ input } \\
\cline { 2 - 5 } & 00 & 01 & 10 & 11 \\
\hline 0000 & 07 & 34 & 62 & 51 \\
\hline 0001 & 34 & 07 & 51 & 62 \\
\hline 0010 & 25 & 16 & 43 & 70 \\
\hline 0011 & 16 & 25 & 70 & 43 \\
\hline 0100 & 61 & 13 & 54 & 26 \\
\hline 0101 & 13 & 61 & 26 & 54 \\
\hline 0110 & 23 & 15 & 52 & 64 \\
\hline 0111 & 15 & 23 & 64 & 52 \\
\hline 1000 & 70 & 43 & 16 & 25 \\
\hline 1001 & 43 & 70 & 25 & 16 \\
\hline 1010 & 51 & 62 & 34 & 07 \\
\hline 1011 & 62 & 51 & 07 & 34 \\
\hline 1100 & 64 & 52 & 15 & 23 \\
\hline 1101 & 52 & 64 & 23 & 15 \\
\hline 1110 & 26 & 54 & 13 & 61 \\
\hline 1111 & 54 & 26 & 61 & 13 \\
\hline
\end{tabular}

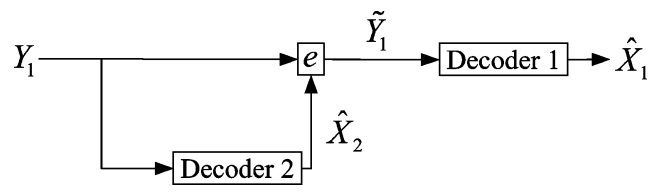

Fig. 11. Decoder structure for user 1.

Receiver 1 uses successive decoding as shown in Fig. 11. Denote as $\hat{X}_{2}$ the decoded stream corresponding to user 2 . Since 


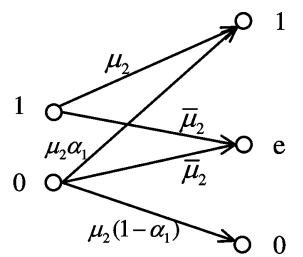

(a) User 1: $\mathrm{Z}$ channel with erasures

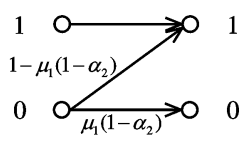

(b) User 2: Z channel
Fig. 12. Perceived channel by each decoder.

the transmitted data is $x=x_{1}(\mathrm{OR}) x_{2}$, whenever a bit $x_{2}=1$, there is no information about $x_{1}$, and $x_{1}$ can be considered an erasure. Hence, the input stream to Decoder 1 is

$$
\hat{y}_{1}=e\left(y_{1}, \hat{x}_{2}\right)= \begin{cases}y_{1}, & \text { if } \hat{x}_{2}=0 \\ e, & \text { if } \hat{x}_{2}=1 .\end{cases}
$$

Therefore, Decoder 2 sees a Z Channel with erasures as shown in Fig. 12. The tools presented in [13] were general enough to be applied to the $\mathrm{Z}$ Channel with erasures. Note that if $\alpha_{1}$ is much smaller than $\alpha_{2}$ we can use hard decoding in Decoder 2 instead of soft decoding without any loss in performance. Since the code for user 2 is designed for a $\mathrm{Z}$ Channel with 0 -to- 1 crossover probability $1-\left(1-\alpha_{2}\right) \mu_{1}$, and the channel perceived by Decoder 2 in user 1 is a Z-Channel with crossover probability $1-\left(1-\alpha_{1}\right) \mu_{1}<1-\left(1-\alpha_{2}\right) \mu_{1}$, the bit error rate of $\hat{x}_{2}$ is negligible compared to the bit error rate of Decoder 1. In fact, in all the simulations shown in Section V, which include 100 frame errors of user 1, none of the errors were produced by Decoder 2 .

\section{Results}

We simulate the transmission strategy for the two-user broadcast $\mathrm{Z}$ channel with crossover probabilities $\alpha_{1}=0.15$ and $\alpha_{2}=0.6$, using nonlinear turbo codes, with the structure shown in Fig. 10. Fig. 13 shows the capacity region for the broadcast $Z$ channel and identifies the simulated rate pairs. It also shows the optimal rate pairs, which are used to compute the ones densities of each code. The output labels for the codes with each simulated rate pair are listed at [15]. For each of these four simulated rate pairs, the loss in mutual information from the associated optimal rate is only 0.04 bits or less in $R_{1}$ and only 0.02 bits or less in $R_{2}$. Table II shows bit error rates for each rate pair, the ones densities $\bar{\mu}_{1}$ and $\bar{\mu}_{2}$, and the interleaver lengths $K_{1}$ and $K_{2}$ used for each code. For simplicity, we chose $K_{1}$ and $K_{2}$ so that the codeword length $n$ would be the same for user 1 and user 2, except for rate pairs $R_{1}=1 / 2$ and $R_{2}=1 / 22$, where one codeword length of user 2 is twice the length of user 1 .

\section{CONCLUSION}

This paper presented an optimal transmission strategy for the broadcast $\mathrm{Z}$ channel with independent encoding and successive decoding. We proved that any point on the optimal boundary of the capacity region can be achieved by independently encoding the messages corresponding to different users and transmitting the OR of the encoded signals. Also, the distributions of the outputs of each encoder that achieve the optimal boundary were provided. Nonlinear-turbo codes that provide a controlled distribution of ones and zeros in their codewords were used to demonstrate a low-complexity scheme that works close to the optimal boundary.

\section{APPENDIX A}

Here we prove Theorem 3 , which states that for a broadcast $\mathrm{Z}$ channel with $0<\alpha_{1}<\alpha_{2}<1$, any transmission strategy $\left(\mu_{1}, \mu_{2}, \gamma\right)$ with $0<\mu_{2}<1,0<\gamma<\mu_{1}$ is not optimal.

In (4) and (5), denote

$$
\begin{aligned}
I_{1}\left(\mu_{1}, \mu_{2}, \gamma\right) & =\left.I\left(X ; Y_{1} \mid X_{2}\right)\right|_{\mu_{1}, \mu_{2}, \gamma} \\
I_{2}\left(\mu_{1}, \mu_{2}, \gamma\right) & =\left.I\left(X_{2} ; Y_{2}\right)\right|_{\mu_{1}, \mu_{2}, \gamma} \\
I_{1,2}\left(\mu_{1}, \mu_{2}, \gamma\right) & =\left.\left(I_{1}, I_{2}\right)\right|_{\mu_{1}, \mu_{2}, \gamma} \cdot
\end{aligned}
$$

The transmission strategy $\left(\mu_{1}, \mu_{2}, \gamma\right)$ achieves the rate pair $I_{1,2}\left(\mu_{1}, \mu_{2}, \gamma\right)$. The theorem is true if we can increase both $I_{1}$ and $I_{2}$ when $0<\mu_{2}<1,0<\gamma<\mu_{1}$.

First compare the strategies $\left(\mu_{1}, \mu_{2}, \gamma\right)$ and $\left(\mu_{1}+\right.$ $\left.\bar{\mu}_{2} \delta_{1}, \mu_{2}, \gamma-\mu_{2} \delta_{1}\right)$ for a small positive number $\delta_{1}>0$

$$
\begin{aligned}
\Delta_{1} I_{1} & =I_{1}\left(\mu_{1}+\bar{\mu}_{2} \delta_{1}, \mu_{2}, \gamma-\mu_{2} \delta_{1}\right)-I_{1}\left(\mu_{1}, \mu_{2}, \gamma\right) \\
& \left.\simeq \frac{\partial I_{1}\left(\mu_{1}+\bar{\mu}_{2} \delta_{1}, \mu_{2}, \gamma-\mu_{2} \delta_{1}\right)}{\partial \delta_{1}}\right|_{\delta_{1}=0} \delta_{1} \\
& =-\mu_{2} \bar{\mu}_{2}\left(1-\alpha_{1}\right)\left\{\ln \frac{1-\gamma\left(1-\alpha_{1}\right)}{\gamma\left(1-\alpha_{1}\right)}\right. \\
& \left.\quad+\ln \frac{\mu_{1}\left(1-\alpha_{1}\right)}{1-\mu_{1}\left(1-\alpha_{1}\right)}\right\} \delta_{1} \\
& <0,
\end{aligned}
$$

and

$$
\begin{aligned}
& \Delta_{1} I_{2}=I_{2}\left(\mu_{1}+\bar{\mu}_{2} \delta_{1}, \mu_{2}, \gamma-\mu_{2} \delta_{1}\right)-I_{2}\left(\mu_{1}, \mu_{2}, \gamma\right) \\
& \simeq\left.\frac{\partial I_{2}\left(\mu_{1}+\bar{\mu}_{2} \delta_{1}, \mu_{2}, \gamma-\mu_{2} \delta_{1}\right)}{\partial \delta_{1}}\right|_{\delta_{1}=0} \delta_{1} \\
&=\mu_{2} \bar{\mu}_{2}\left(1-\alpha_{2}\right)\left\{\ln \frac{1-\gamma\left(1-\alpha_{2}\right)}{\gamma\left(1-\alpha_{2}\right)}\right. \\
&\left.\quad+\ln \frac{\mu_{1}\left(1-\alpha_{2}\right)}{1-\mu_{1}\left(1-\alpha_{2}\right)}\right\} \delta_{1}
\end{aligned}
$$$$
>0 \text {. }
$$

The small change of the rate pair $\left(\Delta_{1} I_{1}, \Delta_{1} I_{2}\right)$ is shown Fig. 14. Point $A$ is the rate pair of the transmission strategy $\left(\mu_{1}, \mu_{2}, \gamma\right)$, the arrow $\Delta_{1}$ shows the small movement of the rate pair $\left(\Delta_{1} I_{1}, \Delta_{1} I_{2}\right)$.

Second compare the strategies $\left(\mu_{1}, \mu_{2}, \gamma\right)$ and $\left(\mu_{1}+(\gamma-\right.$ $\left.\left.\mu_{1}\right) \delta_{2}, \mu_{2}+\mu_{2} \delta_{2}, \gamma\right)$ for a small positive number $\delta_{2}>0$

$$
\begin{aligned}
\Delta_{2} I_{1} & =I_{1}\left(\mu_{1}+\left(\gamma-\mu_{1}\right) \delta_{2}, \mu_{2}+\mu_{2} \delta_{2}, \gamma\right)-I_{1}\left(\mu_{1}, \mu_{2}, \gamma\right) \\
& \left.\simeq \frac{\partial I_{1}\left(\mu_{1}+\left(\gamma-\mu_{1}\right) \delta_{2}, \mu_{2}+\mu_{2} \delta_{2}, \gamma\right)}{\partial \delta_{2}}\right|_{\delta_{2}=0} \delta_{2} \\
& =-\mu_{2} \delta_{2}\left\{\gamma\left(1-\alpha_{1}\right) \ln \frac{\mu_{1}}{\gamma}\right. \\
& \left.\quad+\left(1-\gamma\left(1-\alpha_{1}\right)\right) \ln \frac{1-\mu_{1}\left(1-\alpha_{1}\right)}{1-\gamma\left(1-\alpha_{1}\right)}\right\} \\
& =\mu_{2} \delta_{2} D\left(\gamma\left(1-\alpha_{1}\right) \| \mu_{1}\left(1-\alpha_{1}\right)\right) \\
& >0
\end{aligned}
$$




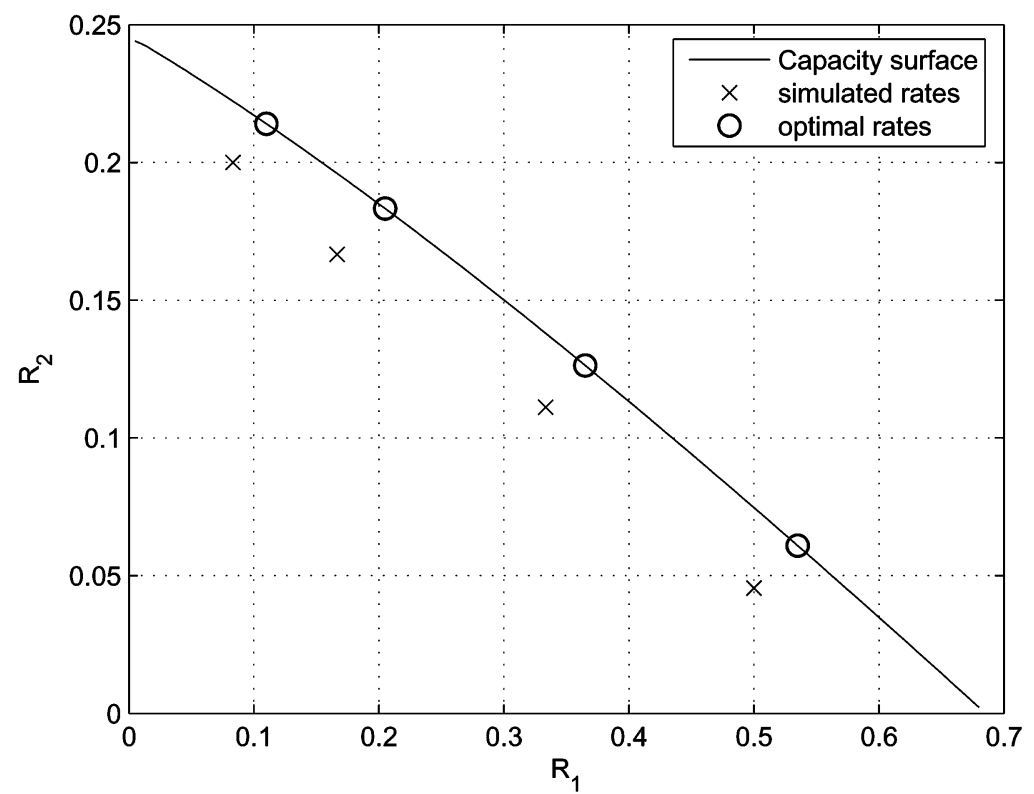

Fig. 13. Broadcast $\mathrm{Z}$ channel with crossover probabilities $\alpha_{1}=0.15$ and $\alpha_{2}=0.6$ for receiver 1 and 2 respectively: achievable capacity region, simulated rate pairs $\left(R_{1}, R_{2}\right)$ and their corresponding optimal rates.

TABLE II

BER For Two-User Broadcast Z Channel With Crossover Probabilities $\alpha_{1}=0.15$ AND $\alpha_{2}=0.6$

\begin{tabular}{|c|c|c|c|c|c|c|c|}
\hline$R_{1}$ & $R_{2}$ & $\bar{\mu}_{1}$ & $\bar{\mu}_{2}$ & $K_{1}$ & $K_{2}$ & BER $_{1}$ & BER $_{2}$ \\
\hline $1 / 12$ & $1 / 5$ & 0.106 & 0.56 & 4800 & 1700 & $2.54 \times 10^{-5}$ & $1.24 \times 10^{-5}$ \\
\hline $1 / 6$ & $1 / 6$ & 0.196 & 0.5 & 2048 & 2048 & $7.01 \times 10^{-6}$ & $5.33 \times 10^{-6}$ \\
\hline $1 / 3$ & $1 / 9$ & 0.336 & 0.3739 & 4608 & 1536 & $7.13 \times 10^{-6}$ & $6.70 \times 10^{-6}$ \\
\hline $1 / 2$ & $1 / 22$ & 0.463 & 0.1979 & 5632 & 1024 & $9.27 \times 10^{-7}$ & $3.27 \times 10^{-6}$ \\
\hline
\end{tabular}

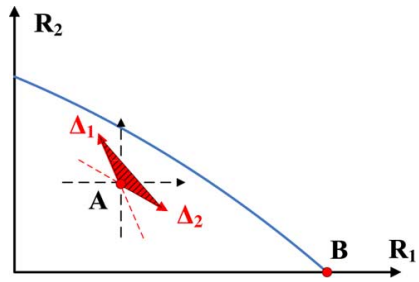

Fig. 14. Capacity region and the changes of rate pairs.

and

$$
\begin{aligned}
\Delta_{2} I_{2} & =I_{2}\left(\mu_{1}+\left(\gamma-\mu_{1}\right) \delta_{2}, \mu_{2}+\mu_{2} \delta_{2}, \gamma\right)-I_{2}\left(\mu_{1}, \mu_{2}, \gamma\right) \\
\simeq & \left.\frac{\partial I_{2}\left(\mu_{1}+\left(\gamma-\mu_{1}\right) \delta_{2}, \mu_{2}+\mu_{2} \delta_{2}, \gamma\right)}{\partial \delta_{2}}\right|_{\delta_{2}=0} \delta_{2} \\
= & \mu_{2} \delta_{2}\left\{\gamma\left(1-\alpha_{2}\right) \ln \frac{\mu_{1}}{\gamma}\right. \\
& \left.\quad+\left(1-\gamma\left(1-\alpha_{2}\right)\right) \ln \frac{1-\mu_{1}\left(1-\alpha_{2}\right)}{1-\gamma\left(1-\alpha_{2}\right)}\right\} \\
& =-\mu_{2} \delta_{2} D\left(\gamma\left(1-\alpha_{2}\right) \| \mu_{1}\left(1-\alpha_{2}\right)\right) \\
<0 &
\end{aligned}
$$

where $D(p \| q)$ is the relative entropy between the distributions $p$ and $q$. The arrow $\Delta_{2}$ in Fig. 14 shows the small movement of the rate pair $\left(\Delta_{2} I_{1}, \Delta_{2} I_{2}\right)$.
Now we show (25)-(26) at the top of the following page.

Let $a=1-\gamma x$ and $b=1-\mu_{1} x$. We have $0<b<a<1$ and want to show that

$$
g(a, b)=\ln \frac{a}{b} \ln \frac{1-a}{1-b}-\left(\ln \frac{a}{b}\right)^{2}+\ln \frac{1-a}{1-b}\left(\frac{1}{a}-\frac{1}{b}\right)>0 .
$$

Since

$$
\frac{\partial^{2} g(a, b)}{\partial a \partial b}=-\frac{(a-b)^{2}}{a^{2} b^{2}(1-a)(1-b)}<0
$$

and

$$
\left.\frac{\partial g(a, b)}{\partial a}\right|_{b=a}=0 \quad \forall 0<a<1
$$

it is true that

$$
\frac{\partial g(a, b)}{\partial a}>0 \quad \forall 0<b<a<1
$$

It follows from (30) and the fact $g(b, b)=0, \forall 0<b<1$ that $g(a, b)>0, \forall 0<b<a<1$. Thus, the inequality (25) is true, which means that the slope of $\Delta_{1}$ is smaller than that of $\Delta_{2}$ in Fig. 14. Hence, the achievable shaded region is on the upper right side of the point $A$. Therefore, we can increase both terms in the rate pair $I_{1,2}\left(\mu_{1}, \mu_{2}, \gamma\right)$ simultaneously and the strategy $\left(\mu_{1}, \mu_{2}, \gamma\right)$ is not optimal when $0<\mu_{2}<1$ and $0<\gamma<\mu_{1}$.

Q.E.D. 


$$
\begin{aligned}
& \frac{\Delta_{1} I_{2}}{\Delta_{1} I_{1}}<\frac{\Delta_{2} I_{2}}{\Delta_{2} I_{1}}<0 \\
& \frac{\Delta_{1} I_{2}}{\Delta_{1} I_{1}}<\frac{\Delta_{2} I_{2}}{\Delta_{2} I_{1}} \\
& \Leftrightarrow \frac{D\left(\gamma\left(1-\alpha_{2}\right) \| \mu_{1}\left(1-\alpha_{2}\right)\right)+\ln \frac{1-\gamma\left(1-\alpha_{2}\right)}{1-\mu_{1}\left(1-\alpha_{2}\right)}}{D\left(\gamma\left(1-\alpha_{1}\right) \| \mu_{1}\left(1-\alpha_{1}\right)\right)+\ln \frac{1-\gamma\left(1-\alpha_{1}\right)}{1-\mu_{1}\left(1-\alpha_{1}\right)}}>\frac{D\left(\gamma\left(1-\alpha_{2}\right) \| \mu_{1}\left(1-\alpha_{2}\right)\right)}{D\left(\gamma\left(1-\alpha_{1}\right) \| \mu_{1}\left(1-\alpha_{1}\right)\right)} \\
& \Leftrightarrow \frac{D\left(\gamma\left(1-\alpha_{1}\right) \| \mu_{1}\left(1-\alpha_{1}\right)\right)}{\ln \frac{1-\gamma\left(1-\alpha_{1}\right)}{1-\mu_{1}\left(1-\alpha_{1}\right)}}>\frac{D\left(\gamma\left(1-\alpha_{2}\right) \| \mu_{1}\left(1-\alpha_{2}\right)\right)}{\ln \frac{1-\gamma\left(1-\alpha_{2}\right)}{1-\mu_{1}\left(1-\alpha_{2}\right)}} \\
& \Leftrightarrow f(x)=\frac{D\left(\gamma x \| \mu_{1} x\right)}{\ln \frac{1-\gamma x}{1-\mu_{1} x}} \text { is monotonically increasing in } 0<x<1 \\
& \Leftrightarrow f^{\prime}(x)=\left\{\ln \frac{\gamma x}{\mu_{1} x} \ln \frac{1-\gamma x}{1-\mu_{1} x}-\left(\ln \frac{1-\gamma x}{1-\mu_{1} x}\right)^{2}+\ln \frac{\gamma x}{\mu_{1} x}\left(\frac{1}{1-\gamma x}-\frac{1}{1-\mu_{1} x}\right)\right\} \gamma\left(\ln \frac{1-\gamma x}{1-\mu_{1} x}\right)^{-2}>0 .
\end{aligned}
$$

$$
\begin{aligned}
\left.\frac{\partial\left(I_{1}+\lambda I_{2}\right)}{\partial \mu_{2}}\right|_{\mu_{2}=1, \mu_{1}=\psi\left(1-\alpha_{1}\right)} & =\left.\frac{\partial I_{1}}{\partial \mu_{2}}\right|_{\mu_{2}=1, \mu_{1}=p s i\left(1-\alpha_{1}\right)}+\left.\lambda \frac{\partial I_{2}}{\partial \mu_{2}}\right|_{\mu_{2}=1, \mu_{1}=\psi\left(1-\alpha_{1}\right)} \\
& \left.\stackrel{\text { (a) }}{<} \frac{\partial I_{1}}{\partial \mu_{2}}\right|_{\mu_{2}=1, \mu_{1}=\psi\left(1-\alpha_{1}\right)}+\left.\varphi\left(\psi\left(1-\alpha_{1}\right)\right) \frac{\partial I_{2}}{\partial \mu_{2}}\right|_{\mu_{2}=1, \mu_{1}=\psi\left(1-\alpha_{1}\right)} \\
& =\left.\frac{\partial\left(I_{1}+\varphi\left(\psi\left(1-\alpha_{1}\right)\right) I_{2}\right)}{\partial \mu_{2}}\right|_{\mu_{2}=1, \mu_{1}=\psi\left(1-\alpha_{1}\right)} \\
& \stackrel{\text { (b) }}{=} 0
\end{aligned}
$$

\section{APPENDIX B}

Here we prove Theorem 4, which provides the unique optimal solution to the maximization problem (12). In problem (12), the objective function $I_{1}+\lambda I_{2}$ is bounded and the domain $0 \leq \mu_{1}$, $\mu_{2} \leq 1$ is closed, so the maximum exists and can be attained. First we discuss some possible optimal solutions and then we show that only one of them is optimal for any fixed $\lambda \geq 0$.

Case 0: If $\mu_{1}=0$ or $\mu_{2}=0$ or $\mu_{1}=\mu_{2}=1$, then $I_{1}=I_{2}=0$ and so it cannot be optimal.

Case 1: If $\mu_{2}=1$ and $0<\mu_{1}<1$, then $I_{2}=0$ and

$$
\begin{aligned}
\frac{\partial I_{1}}{\partial \mu_{1}} & =\left(1-\alpha_{1}\right) \ln \frac{1-\mu_{1}\left(1-\alpha_{1}\right)}{\mu_{1}\left(1-\alpha_{1}\right)}-H\left(1-\alpha_{1}\right) \\
& =0 \\
& \Rightarrow \mu_{1}^{*}=\frac{1}{\left(1-\alpha_{1}\right)\left(e^{H\left(1-\alpha_{1}\right) /\left(1-\alpha_{1}\right)}+1\right)} .
\end{aligned}
$$

Case 2: If $\mu_{1}=1$ and $0<\mu_{2}<1$, then $I_{1}=0$ and

$$
\begin{aligned}
\frac{\partial I_{2}}{\partial \mu_{2}} & =\left(1-\alpha_{2}\right) \ln \frac{1-\mu_{2}\left(1-\alpha_{2}\right)}{\mu_{2}\left(1-\alpha_{2}\right)}-H\left(1-\alpha_{2}\right) \\
& =0 \\
& \Rightarrow \mu_{2}^{*}=\frac{1}{\left(1-\alpha_{2}\right)\left(e^{H\left(1-\alpha_{2}\right) /\left(1-\alpha_{2}\right)}+1\right)} .
\end{aligned}
$$
when

Case 3: If $0<\mu_{1}, \mu_{2}<1$, then the optimum is attained

$$
\begin{gathered}
\mu_{2} \frac{\partial\left(I_{1}+\lambda I_{2}\right)}{\partial \mu_{2}}-\mu_{1} \frac{\partial\left(I_{1}+\lambda I_{2}\right)}{\partial \mu_{1}}=0 \\
\Rightarrow \ln \left(1-\mu_{1}^{*}\left(1-\alpha_{1}\right)\right)=\lambda \ln \left(1-\mu_{1}^{*}\left(1-\alpha_{2}\right)\right)
\end{gathered}
$$

and

$$
\begin{aligned}
\frac{\partial\left(I_{1}+\lambda I_{2}\right)}{\partial \mu_{2}}=0 \\
\Rightarrow \lambda\left\{H\left(\mu_{1}^{*}\left(1-\alpha_{2}\right)\right)-\mu_{1}^{*}\left(1-\alpha_{2}\right) \ln \frac{1-\mu_{2}^{*} \mu_{1}^{*}\left(1-\alpha_{2}\right)}{\mu_{2}^{*} \mu_{1}^{*}\left(1-\alpha_{2}\right)}\right\} \\
=\left(H\left(\mu_{1}^{*}\left(1-\alpha_{1}\right)\right)-\mu_{1}^{*} H\left(1-\alpha_{1}\right)\right) \\
\Rightarrow\left(H\left(\mu_{1}^{*}\left(1-\alpha_{1}\right)\right)-\mu_{1}^{*} H\left(1-\alpha_{1}\right)\right) \cdot \ln \left(1-\mu_{1}^{*}\left(1-\alpha_{2}\right)\right) \\
=\left\{H\left(\mu_{1}^{*}\left(1-\alpha_{2}\right)\right)-\mu_{1}^{*}\left(1-\alpha_{2}\right) \ln \frac{1-\mu_{2}^{*} \mu_{1}^{*}\left(1-\alpha_{2}\right)}{\mu_{2}^{*} \mu_{1}^{*}\left(1-\alpha_{2}\right)}\right\} \\
\quad \cdot \ln \left(1-\mu_{1}^{*}\left(1-\alpha_{1}\right)\right) .
\end{aligned}
$$

For any fixed $\lambda \geq 0$, the optimal solution is in Case 1,2, or 3 .

Lemma 1: Function $\varphi(x)=\frac{\ln \left(1-\left(1-\alpha_{1}\right) x\right)}{\ln \left(1-\left(1-\alpha_{2}\right) x\right)}$ is monotonically increasing in the domain of $0 \leq x \leq 1$ when $\alpha_{1}<\alpha_{2}$.

Lemma 2: The solution in Case 1 cannot be optimal when $\lambda>\varphi\left(\psi\left(1-\alpha_{1}\right)\right)$.

Proof: When $\mu_{2}=1$ and $\mu_{1}=\psi\left(1-\alpha_{1}\right), \frac{\partial I_{2}}{\partial \mu_{1}}=0$ and $\frac{\partial I_{1}}{\partial \mu_{1}}=0$. Therefore, for any fixed $\lambda, \frac{\partial\left(I_{1}+\lambda I_{2}\right)}{\partial \mu_{1}}=0$. When $\lambda=\varphi\left(\mu_{1}\right)=\varphi\left(\psi\left(1-\alpha_{1}\right)\right),(35)$ holds, and so

$$
\begin{aligned}
& \left.\frac{\partial\left(I_{1}+\lambda I_{2}\right)}{\partial \mu_{2}}\right|_{\mu_{2}=1, \mu_{1}=\psi\left(1-\alpha_{1}\right)} \\
= & \left.\frac{\partial\left(I_{1}+\varphi\left(\psi\left(1-\alpha_{1}\right)\right) I_{2}\right)}{\partial \mu_{2}}\right|_{\mu_{2}=1, \mu_{1}=\psi\left(1-\alpha_{1}\right)} \\
= & 0 .
\end{aligned}
$$

When $\lambda>\varphi\left(\psi\left(1-\alpha_{1}\right)\right)$, we get (38) at the top of the page where (a) follows from the facts that $\left.\frac{\partial I_{2}}{\partial \mu_{2}}\right|_{\mu_{2}=1, \mu_{1}=\psi\left(1-\alpha_{1}\right)}=$ 
$\ln \left(1-\psi\left(1-\alpha_{1}\right) \cdot\left(1-\alpha_{2}\right)\right)<0$ and $\lambda>\varphi\left(\psi\left(1-\alpha_{1}\right)\right)$, and (b) follows from (37). Therefore, Case 1 cannot be optimal when $\lambda>\varphi\left(\psi\left(1-\alpha_{1}\right)\right)$.

Q.E.D.

Lemma 3: The solution in Case 2 cannot be optimal when $\lambda<\varphi(1)$.

Proof: When $\mu_{2}=\psi\left(1-\alpha_{2}\right)$ and $\mu_{1}=1, \frac{\partial I_{2}}{\partial \mu_{2}}=0$ and $\frac{\partial I_{1}}{\partial \mu_{2}}=0$. Therefore, for any fixed $\lambda, \frac{\partial\left(I_{1}+\lambda I_{2}\right)}{\partial \mu_{2}}=0$. When $\lambda=\varphi\left(\mu_{1}\right)=\varphi(1),(35)$ holds, and so

$$
\begin{aligned}
& \left.\frac{\partial\left(I_{1}+\lambda I_{2}\right)}{\partial \mu_{1}}\right|_{\mu_{2}=\psi\left(1-\alpha_{2}\right), \mu_{1}=1} \\
& \quad=\left.\frac{\partial\left(I_{1}+\varphi(1) I_{2}\right)}{\partial \mu_{1}}\right|_{\mu_{2}=\psi\left(1-\alpha_{2}\right), \mu_{1}=1} \\
& =0 .
\end{aligned}
$$

When $\lambda<\varphi(1)$

$$
\begin{aligned}
& \left.\frac{\partial\left(I_{1}+\lambda I_{2}\right)}{\partial \mu_{1}}\right|_{\mu_{2}=\psi\left(1-\alpha_{2}\right), \mu_{1}=1} \\
& =\left.\frac{\partial I_{1}}{\partial \mu_{2}}\right|_{\mu_{2}=\psi\left(1-\alpha_{2}\right), \mu_{1}=1}+\left.\lambda \frac{\partial I_{2}}{\partial \mu_{2}}\right|_{\mu_{2}=\psi\left(1-\alpha_{2}\right), \mu_{1}=1} \\
& \left.\quad \stackrel{a}{<} \frac{\partial I_{1}}{\partial \mu_{2}}\right|_{\mu_{2}=\psi\left(1-\alpha_{2}\right), \mu_{1}=1}+\left.\varphi(1) \frac{\partial I_{2}}{\partial \mu_{2}}\right|_{\mu_{2}=\psi\left(1-\alpha_{2}\right), \mu_{1}=1} \\
& =\left.\frac{\partial\left(I_{1}+\varphi(1) I_{2}\right)}{\partial \mu_{1}}\right|_{\mu_{2}=\psi\left(1-\alpha_{2}\right), \mu_{1}=1} \\
& \quad \stackrel{b}{=} 0
\end{aligned}
$$

where (a) follows from the facts that $\left.\frac{\partial I_{2}}{\partial \mu_{1}}\right|_{\mu_{2}=\psi\left(1-\alpha_{2}\right), \mu_{1}=1}=$ $-\psi\left(1-\alpha_{2}\right) \ln \alpha_{2}>0$ and $\lambda<\varphi(1)$, and (b) follows from (39). Therefore, Case 2 cannot be optimal when $\lambda<\varphi(1)$. Q.E.D.

Lemma 4: The solution to (35) exists in $(0,1)$ and is unique for any $\lambda$ in the range of $\varphi(0)<\lambda<\varphi(1)$.

Proof: Equation (35) is equivalent to $\varphi\left(\mu_{1}^{*}\right)=\lambda$. From Lemma $1, \varphi\left(\mu_{1}\right)$ is monotonically increasing. Therefore, when $\varphi(0)<\lambda<\varphi(1)$, the solution $\mu_{1}^{*}$ is unique and $\mu_{1}^{*} \in(0,1)$.

Q.E.D.

Lemma 5: The unique solution $\left(\mu_{1}^{*}, \mu_{2}^{*}\right)$ to (35) and (36) in Case 3 is optimal if $\varphi\left(\psi\left(1-\alpha_{1}\right)\right)<\lambda<\varphi(1)$.

Proof: From Lemma 4, the solution $\mu_{1}^{*}$ to (35) is unique if $\varphi\left(\psi\left(1-\alpha_{1}\right)\right)<\lambda<\varphi(1)$. From (36)

$$
\begin{aligned}
m\left(\mu_{2}\right) & \\
= & \left\{H\left(\mu_{1}^{*}\left(1-\alpha_{2}\right)\right)-\mu_{1}^{*}\left(1-\alpha_{2}\right) \ln \frac{1-\mu_{2} \mu_{1}^{*}\left(1-\alpha_{2}\right)}{\mu_{2} \mu_{1}^{*}\left(1-\alpha_{2}\right)}\right\} \\
& \cdot \ln \left(1-\mu_{1}^{*}\left(1-\alpha_{1}\right)\right) \\
& -\left\{H\left(\mu_{1}^{*}\left(1-\alpha_{1}\right)\right)-\mu_{1}^{*} H\left(1-\alpha_{1}\right)\right\} \\
& \cdot \ln \left(1-\mu_{1}^{*}\left(1-\alpha_{2}\right)\right) \\
= & 0 .
\end{aligned}
$$

Clearly, $m\left(\mu_{2}\right)$ is monotonically increasing,

$$
\lim _{\mu_{2} \rightarrow 0} m\left(\mu_{2}\right)=-\infty<0
$$

and

$$
\begin{aligned}
& \varphi\left(\psi\left(1-\alpha_{1}\right)\right)<\lambda<\varphi(1) \\
& \quad \Rightarrow \mu_{1}^{*}>\psi\left(1-\alpha_{1}\right) \\
& \quad \Rightarrow m(1)>0 .
\end{aligned}
$$

That means the unique solution $\mu_{2}^{*}$ to (36) is in the domain of $0 \leq \mu_{2} \leq 1$. Furthermore, when $\varphi\left(\psi\left(1-\alpha_{1}\right)\right)<\lambda<\varphi(1)$, by Lemma 2 and Lemma 3, Case 1 or Case 2 cannot be optimal because

$$
\begin{array}{ll}
\left.\frac{\partial\left(I_{1}+\lambda I_{2}\right)}{\partial \mu_{2}}\right|_{\mu_{2}=1, \mu_{1}=\psi\left(1-\alpha_{1}\right)} & <0 \\
\left.\frac{\partial\left(I_{1}+\lambda I_{2}\right)}{\partial \mu_{1}}\right|_{\mu_{1}=1, \mu_{2}=\psi\left(1-\alpha_{2}\right)}<0 .
\end{array}
$$

Therefore, Case 3 is optimal.

Q.E.D.

Lemma 6: The unique solution $\left(\mu_{2}^{*}=1, \mu_{1}^{*}=\psi\left(1-\alpha_{1}\right)\right)$ in Case 1 is optimal if $0 \leq \lambda \leq \varphi\left(\psi\left(1-\alpha_{1}\right)\right)$.

Proof: When $0 \leq \lambda \leq \varphi\left(\psi\left(1-\alpha_{1}\right)\right)$, Case 3 is not optimal because there is no solution $\mu_{1} \in(0,1)$ to (35). Case 2 is not optimal by Lemma 3 . Hence, Case 1 is optimal.

Q.E.D.

Lemma 7: The unique solution $\left(\mu_{2}^{*}=\psi\left(1-\alpha_{2}\right), \mu_{1}^{*}=1\right)$ in Case 2 is optimal if $\lambda \geq \varphi(1)$.

Proof: When $\lambda \geq \varphi(1)$, Case 3 is not optimal because there is no solution $\mu_{2} \in(0,1)$ to (36). Case 1 is not optimal by Lemma 2. Hence, Case 2 is optimal.

Q.E.D.

From Lemma 5, 6, and 7, Theorem 4 is immediately proved.

Q.E.D.

\section{REFERENCES}

[1] T. M. Cover, "Broadcast channels," IEEE Trans. Inf. Theory, vol. IT-18, pp. 2-14, Jan. 1972.

[2] P. P. Bergmans, "Random coding theorem for broadcast channels with degraded components," IEEE Trans. Inf. Theory, vol. IT-19, pp. 197-207, Mar. 1973.

[3] P. P. Bergmans, "A simple converse for broadcast channels with additive white Gaussian noise," IEEE Trans. Inf. Theory, vol. IT-20, pp. 279-280, Mar. 1974

[4] R. G. Gallager, "Capacity and coding for degraded broadcast channels," Probl. Pered. Inform., vol. 10, pp. 3-14, Jul.-Sep. 1974.

[5] T. M. Cover, "Comments on broadcast channels," IEEE Trans. Inf. Theory, vol. 44, pp. 2524-2530, Oct. 1998.

[6] C. Berrou, A. Glavieux, and P. Thitimajshima, "Near shannon limit error-correcting coding and decoding: Turbo-codes," in Proc. ICC'93, May 1993, pp. 873-890.

[7] R. G. Gallager, "Low-Density Parity-Check Codes," Ph.D. dissertation, Massachusetts Institute of Technology, Cambridge, MA, 1963.

[8] P. Berlin and D. Tuninetti, "LDPC codes for Gaussian broadcast channels," in Proc. 2004 IEEE 5th Workshop on Signal Process., Advances in Wireless Commun., , 2004, pp. 444-448.

[9] T. W. Sun, R. D. Wesel, M. R. Shane, and K. Jarett, "Superposition turbo-TCM for multi-rate broadcast," IEEE Trans. Commun., vol. 52, pp. 368-371, 2004.

[10] P. R. Chevillat, "N-user trellis coding for a class of multiple-access channels," IEEE Trans. Inf. Theory, vol. IT-27, pp. 114-120, 1981.

[11] S. W. Golomb, "The limiting behavior of the Z-channel," IEEE Trans. Inf. Theory, vol. IT-26, pp. 372-372, May 1980.

[12] M. Griot, A. I. V. Casado, W.-Y. Weng, H. Chan, J. Basak, E. Yablanovitch, I. Verbauwhede, B. Jalali, and R. D. Wesel, "Trellis codes with low ones density for the OR multiple access channel," in Proc. IEEE ISIT 2006, Jul. 2006.

[13] M. Griot, A. I. V. Casado, and R. D. Wesel, "Non-linear turbo codes for interleaver-division multiple access on the or channel," in Proc. IEEE GLOBECOM'06 Conf., Nov.-Dec. 2006.

[14] M. Griot, A. I. V. Casado, W.-Y. Weng, H. Chan, and R. D. Wesel, "Nonlinear trellis codes for binary-input binary-output multiple access channels with single-user decoding," IEEE Trans. Commun. .

[15] Nonlinear Turbo Codes for Broadcast Z Channels [Online]. Available: http://www.ee.ucla.edu/ csl/files/codes/bzc.html 